\title{
Compact and accurate variational wave functions of three-electron atomic systems constructed from semi-exponential radial basis functions
}

\author{
Alexei M. Frolov* \\ Department of Chemistry \\ University of Western Ontario, \\ London, Ontario N6H 5B\%, Canada
}

(Dated: May 29, 2022)

\begin{abstract}
The semi-exponential basis set of radial functions (A.M. Frolov, Physics Letters A 374, $2361(2010))$ is used for variational computations of bound states in three-electron atomic systems. It appears that semi-exponential basis set has a substantially greater potential for accurate variational computations of bound states in three-electron atomic systems than it was originally anticipated. In particular, the 40-term Larson's wave function improved with the use of semi-exponential radial basis functions now produces the total energy -7.47805413551 a.u. for the ground $1^{2} S$-state in the ${ }^{\infty} \mathrm{Li}$ atom (only one spin function $\chi_{1}=$ $\alpha \beta \alpha-\beta \alpha \alpha$ was used in these calculations). This variational energy is very close to the exact ground state energy of the ${ }^{\infty} \mathrm{Li}$ atom and it substantially lower than the total energy obtained with the original Larson's 40-term wave function (-7.477944869 a.u.).

PACS number(s): 03.65.Ge, 31.15.ac and 31.15.xf
\end{abstract}

${ }^{*}$ E-mail address: afrolov@uwo.ca 


\section{INTRODUCTION}

In this study we perform variational calculations of bound states in three-electron atomic systems. An advanced basis set of semi-exponential radial functions [2] is extensively used in our calculations. The main goal is to solve the non-relativistic Schrödinger equation $H \Psi=E \Psi$, where $E<0$ and bound state wave function $\Psi$ has the unit norm. The general non-relativistic Hamiltonian $H$ of the three-electron atomic problem is (see, e.g., [1])

$$
H=-\frac{\hbar^{2}}{2 m_{e}}\left[\nabla_{1}^{2}+\nabla_{2}^{2}+\nabla_{3}^{2}+\frac{m_{e}}{M} \nabla_{4}^{2}\right]-\frac{Q e^{2}}{r_{14}}-\frac{Q e^{2}}{r_{24}}-\frac{Q e^{2}}{r_{34}}+\frac{e^{2}}{r_{12}}+\frac{e^{2}}{r_{13}}+\frac{e^{2}}{r_{23}}
$$

where $\hbar=\frac{h}{2 \pi}$ is the reduced Planck constant, $m_{e}$ is the electron mass and $e$ is the electric charge of electron. In this equation and everywhere below in this study the subscripts 1, 2, 3 designate the three electrons $e^{-}$, while the subscript 4 denotes the heavy nucleus with the mass $M\left(M \gg m_{e}\right)$ and positive electric (nuclear) charge $Q e$. The notations $r_{i j}=\left|\mathbf{r}_{i}-\mathbf{r}_{j}\right|=r_{j i}$ stand for the six interparticle distances (= relative coordinates) defined in an arbitrary four-body system and $\mathbf{r}_{i}(i=1,2,3,4)$ are the Cartesian coordinates of the four point particles. In Eq.(1) and everywhere below in this work we shall assume that $(i j)=(j i)=(12),(13),(14),(23),(24),(34)$. In fact, below only atomic units $\hbar=1,|e|=1, m_{e}=1$ are employed. In these units the explicit form of the Hamiltonian $H$, Eq.(1), is significantly simplified.

The main attention in this work is focused on numerical calculations of the ground (doublet) $1^{2} S(L=0)$-state (or $1^{2} S_{\frac{1}{2}}(L=0)$-state) of the three-electron Li atom with the infinitely heavy nucleus, i.e. the ${ }^{\infty} \mathrm{Li}$ atom. Considerations of other three-electron atoms, ions and various positron containing atomic systems (e.g., HPs) can be performed absolutely analogously (for more detail, see [2]) and, therefore, these systems will not be considered here.

The problem of highly accurate calculations of the bound states in three-electron atomic systems has attracted a very significant attention. First calculations of the Li atom with the truly correlated wave functions were performed in 1936 [3]. A brief reviews of such calculations can be found in [4] (earlier works) and [5] (references up to 1997 are mentioned). The current bibliography on this subject includes almost one thousand references and rapidly increases. At this moment the classical Hylleraas method (see, e.g., [5]) is only the method used to construct highly accurate wave functions for bound states in three-electron atomic 
systems. In this method to produce the highly accurate wave functions, e.g., for the ground (doublet) $1^{2} S$-state in the Li atom one needs to use many thousands Hylleraas basis functions. The use of extremely large basis sets is very inconvenient in many actual cases, since it produces a number of computational problems. It is clear that the Hylleraas method cannot be used to construct compact and accurate variational wave functions for three-electron systems. Indeed, it contains essentially no control parameters which can be optimized by increasing the overall efficiency of the method.

An alternative approach to variational bound state calculations in three-electron atomic systems was proposed in [2]. This approach is based on the use of semi-exponential variational wave functions [2] and it allows one to construct very compact and accurate variational wave functions in arbitrary three-electron atomic system. Each of the semi-exponential basis functions depend upon all six interparticle coordinates $r_{12}, r_{13}, \ldots, r_{34}$ [2]. A very high efficiency of this new approach in actual applications and its superiority over the classical Hylleraas expansion was demonstrated in [2]. As follows from the results of this study the semi-exponential basis set has a substantially greater potential for highly accurate variational computations of bound states in three-electron atomic systems than it was anticipated earlier [2].

\section{VARIATIONAL WAVE FUNCTION}

The variational wave function of the doublet $S(L=0)$-states of the three-electron Li atom is written in the following general form

$$
\Psi_{L=0}=\psi_{L=0}\left(A ;\left\{r_{i j}\right\}\right)(\alpha \beta \alpha-\beta \alpha \alpha)+\phi_{L=0}\left(B ;\left\{r_{i j}\right\}\right)(2 \alpha \alpha \beta-\beta \alpha \alpha-\alpha \beta \alpha)
$$

where $\psi_{L=0}\left(A ;\left\{r_{i j}\right\}\right)$ and $\phi_{L=0}\left(B ;\left\{r_{i j}\right\}\right)$ are the two independent spatial parts (= radial parts) of the total wave function. Each of these two radial functions is, in fact, a radial factor (for states with $L=0$ ) in front of the corresponding three-electron spin functions $\chi_{1}=\alpha \beta \alpha-\beta \alpha \alpha$ and $\chi_{2}=2 \alpha \alpha \beta-\beta \alpha \alpha-\alpha \beta \alpha$. Here the notations $\alpha$ and $\beta$ are the oneelectron spin-up and spin-down functions, respectively (see, e.g., [6]). The notations $A$ and $B$ in Eq.(2) mean that the two sets of non-linear parameters associated with radial functions $\psi$ and $\phi$ can be optimized independently. In the general case, each of the radial basis functions explicitly depends upon all six interparticle (relative) coordinates $r_{12}, r_{13}, r_{23}, r_{14}, r_{24}, r_{34}$. It 
is clear that in actual variational calculations only one spin function, e.g., the $\chi_{1}$ function, can be used. Another useful trick (so-called 'doubling') is based on the use of the same set of non-linear parameters in the two radial parts in Eq.(2).

In our earlier work [2] we have introduced an advanced set of radial basis functions for three-electron atomic calculations. In [2] this set was called the semi-exponential basis set. In general, the semi-exponential variational expansion of the radial function $\psi_{L=0}\left(A ;\left\{r_{i j}\right\}\right)$ is written in the form

$$
\psi_{L=0}\left(A ;\left\{r_{i j}\right\}\right)=\sum_{k=1}^{N} C_{k} r_{23}^{n_{1}(k)} r_{13}^{n_{2}(k)} r_{12}^{n_{3}(k)} r_{14}^{m_{1}(k)} r_{24}^{m_{2}(k)} r_{34}^{m_{3}(k)} \exp \left(-\alpha_{k} r_{14}-\beta_{k} r_{24}-\gamma_{k} r_{34}\right)
$$

where $\alpha_{k}, \beta_{k}, \gamma_{k}(k=1,2, \ldots, N)$ are the varied non-linear parameters. The presence of the varied non-linear parameters in Eq.(3) is the main and very important difference with the traditional Hylleraas variational expansion (see, e.g., [7]) for which in Eq.(33) we always have $\alpha_{1}=\ldots=\alpha_{N}, \beta_{1}=\ldots=\beta_{N}$ and $\gamma_{1}=\ldots=\gamma_{N}$. Note that all matrix elements of the Hamiltonian, Eq.(1), and overlap matrix needed in computations with the use of the semi-exponential basis, Eq.(3), contain the same three-electron integrals which arise for the usual Hylleraas expansion (for more detail, see [2]). In other words, numerical calculation of all matrix elements with our semi-exponential functions is no more difficult problem, than for the traditional Hylleraas radial functions. This also simplifies numerical computation of the bound state properties (i.e. expectation values) in the semi-exponential basis set. Our algorithms used in calculations of all required matrix elements is based on the Perkins formula for three-electron integrals [8] in relative coordinates. Note also that all calculations in this work have been performed with the use of standard quadruple precision accuracy (30 decimal digits per computer word).

In actual atomic systems any many-electron wave function must be completely antisymmetric upon all electron variables, i.e. upon all electron spatial and spin variables. For three-electron atomic wave function this requirement is written in the form $\hat{\mathcal{A}}_{123} \Psi(1,2,3)=-\Psi(1,2,3)$, where $\Psi$ is given by Eq.(2) and $\hat{\mathcal{A}}_{e}$ is the three-particle $(=$ electron) antisymmetrizer $\hat{\mathcal{A}}_{e}=\hat{e}-\hat{P}_{12}-\hat{P}_{13}-\hat{P}_{23}+\hat{P}_{123}+\hat{P}_{132}$. Here $\hat{e}$ is the identity permutation, while $\hat{P}_{i j}$ is the permutation of the $i$-th and $j$-th particles. Analogously, the operator $\hat{P}_{i j k}$ is the permutation of the $i$-th, $j$-th and $k$-th particles. In actual computations antisymmetrization of the total wave function is reduced to the proper antisymmetrization of corresponding matrix elements (for more detail, see, e.g., [2]). Each of these matrix ele- 
ments is written in the form $\langle\Psi|\hat{O}| \Psi\rangle$, where $\hat{O}$ is an arbitrary spin-independent quantum operator which is truly symmetric upon all interparticle permutations. The wave function $\Psi$, Eq.(2), contains the two different radial parts $\psi$ and $\phi$. By performing the integration over all spin coordinates from here one finds the four spatial projectors $\mathcal{P}_{\psi \psi}, \mathcal{P}_{\psi \phi}=\mathcal{P}_{\phi \psi}$ and $\mathcal{P}_{\phi \phi}$ presented in [2]. In fact, the explicit form of the $\mathcal{P}_{\psi \phi}$ and $\mathcal{P}_{\phi \psi}$ projectors given in [2] must be corrected (there is an obvious misprint in the formulas given in [2])

$$
\begin{aligned}
& \mathcal{P}_{\psi \phi}=\frac{1}{2}\left(\hat{P}_{13}-\hat{P}_{23}+\hat{P}_{123}-\hat{P}_{132}\right) \\
& \mathcal{P}_{\phi \psi}=\frac{1}{2}\left(\hat{P}_{13}-\hat{P}_{23}+\hat{P}_{123}-\hat{P}_{132}\right)
\end{aligned}
$$

For an arbitrary truly symmetric spin-independent operator $\hat{O}$ each of these four projectors produces matrix elements $\langle\Psi|\hat{O}| \Psi\rangle$ of the correct permutation symmetry (for doublet states) between all three electrons. The explicit formulas for all matrix elements obtained with the radial basis functions, Eq.(3) , and for three-electron integrals needed in calculations can be found in [2].

\section{CALCULATIONS}

Let us apply the semi-exponential variational expansion, Eq.(3), to numerical calculations of the ground $1^{2} S$-state in the three-electron ${ }^{\infty} \mathrm{Li}$ atom. In this study we consider the two variational wave functions: (a) the wave function which contains 28 radial basis functions, Eq.(3) , and (b) the wave function which includes 40 radial basis functions, Eq.(3)). The results (in atomic units) obtained with these two trial wave functions can be found in Table I. Tables II and III contain the corresponding radial basis functions, Eq.(3), i.e. the powers $n_{1}(k), n_{2}(k), n_{3}(k), m_{1}(k), m_{2}(k), m_{3}(k)$ of six radial variables $r_{12}, r_{13}, r_{23}, r_{14}, r_{24}, r_{34}$ and optimized non-linear parameters $\alpha_{k}, \beta_{k}, \gamma_{k}$. As follows from Table I our variational energies obtained for the ground $1^{2} S$-state in the ${ }^{\infty} \mathrm{Li}$ atom with the use of semi-exponential variational expansion, Eq.(2), are substantially lower than the corresponding energies determined for this state with the same Hylleraas wave function [7]. Note also that the non-linear parameters used in our method (in Eq.(31) ) are constantly varied. Therefore, it is hard to say that the total energies obtained in some calculations are 'final'. Formally, based on the known convergence rate(s) for our data and by using a few extrapolation procedures we can approximately evaluate the limits to which our variational energies will converge, if we 
could perform an infinite number of variations for the non-linear parameters in Eq.(3). Such limits for the total energies are shown in the fourth column of Table I. These values indicate that, e.g., our 40-term variational wave function can produce, in principle, very accurate variational energies, if the optimization of non-linear parameters in Eq.(3) will continue.

In [7] Larsson proposed a simple (but useful!) trick which allows one to increase the overall accuracy of the trial (doublet) wave function. Later this trick was called 'doubling' of the wave function and it was used practically in all calculations of the bound doublet states in three-electron atomic systems. The idea of doubling is simple and transparent. If we already know the radial function constructed for one spin configuration, e.g., for $\chi_{1}=\alpha \beta \alpha-\beta \alpha \alpha$ from Eq.(2), then we can use exactly the same radial basis function for another spin configuration $\chi_{2}=2 \alpha \alpha \beta-\beta \alpha \alpha-\alpha \beta \alpha$. Formally, it doubles the total number of basis functions in the trial wave function. According to the variational principle the total variational energy can only decrease during such a procedure. The problem of linear dependence of basis functions is avoided in this procedure, since the two spin functions $\chi_{1}=\alpha \beta \alpha-\beta \alpha \alpha$ and $\chi_{2}=2 \alpha \alpha \beta-\beta \alpha \alpha-\alpha \beta \alpha$ are independent of each other. In fact, for Hylleraas variational expansion the 'doubling' does not work properly, since there are obvious linear dependencies between different radial basis functions in those cases when some non-linear parameters coincide with each other (for more detail, see [7]). In semi-exponential variational expansion Eq.(3) all optimized non-linear parameters are independent of each other. Therefore, the coincidence of the pre-exponential factors in Eq.(3) is not crucial and does not mean that such basis functions are linearly dependent. This drastically simplifies the actual 'doubling' for Eq.(3) . The energies obtained with the use of 'doubling' of our variational wave functions can be found in the fifth column of Table I. It is clear that the non-linear parameters (or parameters in the exponents in Eq.(3) ) from the second part of the total wave function are not optimal, i.e. for all terms which contain basis functions with numbers $i \geq 41$ the non-linear parameters are not optimal. These $120(=40 \times 3)$ non-linear parameters can be re-optimized and this drastically improves the overall quality of the total wave function. For instance, approximate one-step re-optimization of the last 40 non-linear parameters in the wave function gives the ground state energy -7.47805456511 a.u., which is much better than the 'doubling' energy from Table I.

As follows from Table I the doubling is not an effective approach for our trial wave functions with the carefully optimized non-linear parameters. However, we can modify the 
original idea of doubling into something new which is substantially more effective in actual computations. To illustrate one of such modifications, let us assume that we have constructed 40 -term variational wave function, Eq.(3) which contains $40 \times 3=120$ carefully optimized non-linear parameters $\alpha_{1}, \beta_{1}, \gamma_{1}, \ldots, \alpha_{40}, \beta_{40}, \gamma_{40}$. At the second step of our procedure we can add forty additional basis functions with the same pre-exponential factors $r_{23}^{n_{1}(k)} r_{13}^{n_{2}(k)} r_{12}^{n_{3}(k)} r_{14}^{m_{1}(k)} r_{24}^{m_{2}(k)} r_{34}^{m_{3}(k)}$, but slightly different exponents in Eq.(3)). In reality, these new exponents have been chosen quasi-randomly from three different intervals, e.g.,

$$
\begin{gathered}
\alpha_{i+40}=\alpha_{i}+0.0057 \cdot\left\langle\left\langle\frac{i(i+1) \sqrt{2}}{2}\right\rangle\right\rangle \\
\beta_{i+40}=\beta_{i}+0.0063 \cdot\left\langle\left\langle\frac{i(i+1) \sqrt{3}}{2}\right\rangle\right\rangle \\
\gamma_{i+40}=\gamma_{i}+0.0049 \cdot\left\langle\left\langle\frac{i(i+1) \sqrt{5}}{2}\right\rangle\right\rangle
\end{gathered}
$$

where $i=1,2, \ldots, 40$ and $\langle\langle x\rangle\rangle$ designates the fractional part of the real number $x$. Small deviations of these new exponents from the known 'optimal' values (i.e. from $\alpha_{i}, \beta_{i}, \gamma_{i}$, where $1 \leq i \leq 40)$ produce the extended wave function of 'almost optimal' quality. On the other hand, even these small differences between exponents allows one to avoid a linear dependence between basis vectors in Eq.(3). Obviously, this procedure can be repeated a number of times. This allows one to construct very accurate trial wave functions which contain not only 80 , but 400, 800 and even 2000 basis functions with almost 'optimal' nonlinear parameters.

It is very interesting to perform variational calculations of the ground state of the ${ }^{\infty} \mathrm{Li}$ atom with the use of 60-term wave function constructed from the analogous 60-term wave Larsson's wave function [7]. The variational total energy obtained in [7] with that wave function was -7.47801035965 a.u. Our 60-term trial wave function with one spin function $\chi_{1}$ constructed in [2] corresponds to the substantially lower total energy -7.478057561 a.u. After publication of [2] we have started re-optimization of the non-linear parameters in our 60-term trial wave function. The current total energy is -7.47805637319 a.u. (only one spin function $\chi_{1}$ is used in our calculations). This energy is above the value from [2]. However, our current total energy rapidly decreases with almost constant rate $\approx 2.5 \cdot 10^{-7}$ a.u. per cycle, i.e. per one variation of all $180(=60 \times 3)$ non-linear parameters in the trial wave function. We expect that after an infinite number of variations of the non-linear parameters the total energy of the ${ }^{\infty} \mathrm{Li}$ atom obtained with our 60 -term radial function and one spin function 
will converge to the value $-7.4780603(3) a . u$. which is very close to the actual ground state energy. It will be an outstanding result to obtain the value lower than -7.4780602 a.u. for the total energy of the ground $1^{2} S$-state in the ${ }^{\infty} \mathrm{Li}$ atom by using only 60 -term variational wave function.

\section{CONCLUSION}

The semi-exponential variational expansion [2] is applied for bound state calculations of three-electron atomic systems. It is shown that this variational expansion allows one to construct very compact and accurate variational wave functions for three-electron atomic systems. Currently, the use of semi-exponential radial basis functions is the only way to produce compact and accurate wave functions for three-electron atomic systems. The total energies obtained in this study for the ground $1^{2} S$-state of the ${ }^{\infty} \mathrm{Li}$ atom (see Table I) are more accurate than our earlier results from [2] and substantially more accurate than the original Larsson's wave function [7] with the same number of terms. It indicates clearly that our semi-exponential variational expansion, Eq.(3) , has a substantially greater potential for variational bound state calculations in three-electron atomic systems than we have

anticipated originally [2]. Currently, we continue the process of numerical optimization of the non-linear parameters in our trial wave functions constructed with the use of semiexponential variational expansion. Note that the choice of Larsson's wave function(s) as the first approximation to the semi-exponential variational expansion is not crucial for our method. Many other (different) choices are also possible.

The semi-exponential variational expansion is also applied for accurate computations of various atomic and quasi-atomic three-electron systems. In particular, we have made numerous improvements in our original computer code [2]. It is clear that the semi-exponential basis can be used to construct variational wave functions in the four-electron atomic problems. In particular, the semi-exponential variational expansion can be used to obtain very compact and accurate wave functions of the ground singlet $1^{1} S$-state and triplet $2^{3} S$-states of the Be atom and Be-like ions (for more details, see [9], [10] and reference therein). It is clear that our method can also be used for rotationally excited states in atomic systems, i.e. 
for states with $L \geq 1$, where $L$ is the electron angular momentum.

[1] L.D. Landau and E.M. Lifshitz, Quantum Mechanics. Non-Relativistic Theory, (3rd. ed., Oxford, England, Pergamon Press (1977)).

[2] A.M. Frolov, Phys. Lett. A 374, 2361 (2010).

[3] H. James and A.S. Coolidge, Phys. Rev. 49, 688 (1936).

[4] R. McWeeny and B.T. Satcliffe, Methods of Molecular Quantum Mechanics, (New York: Academic Press, 1969), Chp. 7.

[5] F.W. King, J. Mol. Struc. (Theochem) 400, 7 (1997).

[6] P.A.M. Dirac, Principles of Quantum Mechanics, (Oxford: Clarendon Press, 4th Ed., 1958).

[7] S. Larsson, Phys. Rev. 169, 49 (1968).

[8] J.F. Perkins, J. Chem. Phys. 48, 1985 (1968).

[9] J. Sims and S.A. Hagstrom, Phys. Rev. A 4, 908 (1971).

[10] A.M. Frolov and D.M. Wardlaw, Phys. Rev. A 79, 064501 (2009). 
TABLE I: The total energies $E$ (in atomic units) of the $1^{2} S(L=0)$-state in the ${ }^{\infty} \mathrm{Li}$ atom. $N$ designates the number of basis functions used.

\begin{tabular}{|c|c|c|c|}
\hline$N E$ (Ref.[4]) & $E$ (Eq. $(3))$ & $E^{a}($ Eq. $(3))$ & $E$ (Eq.(3), doubling) \\
\hline $28-7.477885105$ & -7.47803638005 & $-7.4780368(3)$ & -7.47803657859 \\
\hline $40-7.477944869$ & -7.47805413551 & $-7.4780575(10)$ & -7.47805422814 \\
\hline
\end{tabular}


TABLE II: Semi-exponential radial basis functions for $N=28$ with optimized exponents. This wave function produces the total energy $E=-7.47803638005$ a.u. for the ground $1^{2} S$-state of the ${ }^{\infty} \mathrm{Li}$ atom. Only one electron spin-function $\chi_{1}=\alpha \beta \alpha-\beta \alpha \alpha$ was used in these calculations.

\begin{tabular}{|c|c|c|c|c|c|c|c|c|c|}
\hline$N$ & & & & & & & $\alpha$ & $\beta$ & $\gamma$ \\
\hline 1 & 0 & 0 & 0 & 0 & 0 & 1 & $0.340570905705403 \mathrm{E}+01$ & $0.293295186982955 \mathrm{E}+01 \mathrm{c}$ & 0.77154618823110 \\
\hline 2 & 0 & 0 & 0 & 1 & 0 & 1 & $0.182145794896563 \mathrm{E}+01$ & $0.329011437023099 \mathrm{E}+01 \mathrm{c}$ & $0.316360558089102 \mathrm{E}+01$ \\
\hline 3 & 0 & 0 & 0 & 1 & 1 & 1 & $0.276859627151255 \mathrm{E}+01$ & $0.297627929771076 \mathrm{E}+01 \mathrm{c}$ & $0.668289237018917 \mathrm{E}+00$ \\
\hline 4 & 0 & 0 & 0 & 2 & 0 & 1 & $0.286729169596989 \mathrm{E}+01$ & $0.300017942427107 \mathrm{E}+01 \mathrm{c}$ & $0.637008666805219 \mathrm{E}+00$ \\
\hline 5 & 0 & 0 & 1 & 0 & 0 & 1 & $0.278208405003856 \mathrm{E}+01$ & $0.275168552095737 \mathrm{E}+01$ & $0.645451709771134 \mathrm{E}+00$ \\
\hline 6 & 0 & 0 & 2 & 0 & 0 & 1 & $0.373836260739635 \mathrm{E}+01$ & $0.339167728695617 \mathrm{E}+01 \mathrm{c}$ & $0.658338285572628 \mathrm{E}+00$ \\
\hline 7 & 0 & 0 & 0 & 0 & 0 & 0 & $0.337994212648889 \mathrm{E}+01$ & $0.324225523017272 \mathrm{E}+01 \mathrm{c}$ & $0.108564332122329 \mathrm{E}+01$ \\
\hline 8 & 1 & 0 & 0 & 0 & 0 & 0 & $0.167110811817316 \mathrm{E}+01$ & $0.338626357605054 \mathrm{E}+01$ & $0.867142603936635 \mathrm{E}+00$ \\
\hline 9 & 0 & 0 & 0 & 0 & 0 & 2 & $0.306529447275211 \mathrm{E}+01$ & $0.299446682705661 \mathrm{E}+01 \mathrm{c}$ & $0.688809932201360 \mathrm{E}+00$ \\
\hline 10 & 1 & 0 & 0 & 0 & 1 & 0 & $0.263305048764256 \mathrm{E}+01$ & $0.242503640146648 \mathrm{E}+01 \mathrm{c}$ & $0.776895925198369 \mathrm{E}+00$ \\
\hline 11 & 0 & 0 & 3 & 0 & 0 & 1 & $0.521025323497647 \mathrm{E}+01$ & $0.361243051429800 \mathrm{E}+01 \mathrm{c}$ & $0.660062464430887 \mathrm{E}+00$ \\
\hline 12 & 1 & 0 & 0 & 0 & 0 & 1 & $0.306893947287392 \mathrm{E}+01$ & $0.208144332232383 \mathrm{E}+01 \mathrm{c}$ & $0.123105191974125 \mathrm{E}+01$ \\
\hline 13 & 0 & 0 & 0 & 0 & 0 & 3 & $0.186312473874842 \mathrm{E}+01$ & $0.289718514568496 \mathrm{E}+01$ & $0.996173732965274 \mathrm{E}+00$ \\
\hline 14 & 0 & 0 & 1 & 1 & 0 & 1 & $0.454831261352940 \mathrm{E}+01$ & $0.219828523532609 \mathrm{E}+01$ & $0.158185067718071 \mathrm{E}+01$ \\
\hline 15 & 0 & 0 & 0 & 3 & 0 & 1 & $0.295559769377747 \mathrm{E}+01$ & $0.327296616683230 \mathrm{E}+01$ & $0.672095727321017 \mathrm{E}+00$ \\
\hline 16 & 0 & 0 & 4 & 0 & 0 & 1 & $0.113723992478698 \mathrm{E}+02$ & $0.508008324231496 \mathrm{E}+01 \mathrm{c}$ & $0.636162427376743 \mathrm{E}+00$ \\
\hline 17 & 0 & 0 & 0 & 2 & 2 & 1 & $0.368595304619283 \mathrm{E}+01$ & $0.278359204737422 \mathrm{E}+01$ & $0.620935860917418 \mathrm{E}+00$ \\
\hline 18 & 0 & 0 & 1 & 1 & 1 & 1 & $0.357466607750891 \mathrm{E}+01$ & $0.343630387369804 \mathrm{E}+01 \mathrm{c}$ & $0.698563354607838 \mathrm{E}+00$ \\
\hline 19 & 0 & 0 & 1 & 2 & 0 & 1 & $0.365095130448352 \mathrm{E}+01$ & $0.317945977760753 \mathrm{E}+01$ & $0.652269908234656 \mathrm{E}+00$ \\
\hline 20 & 0 & 0 & 1 & 3 & 0 & 1 & $0.290005170216145 \mathrm{E}+01$ & $0.289825235917765 \mathrm{E}+01$ & $0.685753232846547 \mathrm{E}+00$ \\
\hline 21 & 2 & 0 & 0 & 0 & 0 & 0 & $0.280340788922781 \mathrm{E}+01$ & $0.225117021991187 \mathrm{E}+01$ & $0.990926897297406 \mathrm{E}+00$ \\
\hline 22 & 1 & 1 & 0 & 0 & 0 & 0 & $0.275272575017714 \mathrm{E}+01$ & $0.276113990248958 \mathrm{E}+01$ & $0.802457922378512 \mathrm{E}+00$ \\
\hline 23 & 1 & 0 & 0 & 0 & 2 & 0 & $0.606571100003296 \mathrm{E}+01$ & $0.326870035708320 \mathrm{E}+01$ & $0.453868977046091 \mathrm{E}+00$ \\
\hline 24 & 1 & 0 & 0 & 1 & 1 & 0 & $0.293743657648302 \mathrm{E}+01$ & $0.226578275529031 \mathrm{E}+01 \mathrm{c}$ & $0.669408905271258 \mathrm{E}+00$ \\
\hline 25 & 0 & 0 & 0 & 0 & 0 & 4 & $0.502952034298549 \mathrm{E}+01$ & $0.276772288773810 \mathrm{E}+01$ & $0.950599883558449 \mathrm{E}+00$ \\
\hline 26 & 0 & 0 & 1 & 0 & 0 & 0 & $0.868992405342042 \mathrm{E}+01$ & $0.374899612568756 \mathrm{E}+01$ & $0.516228416453001 \mathrm{E}+00$ \\
\hline 27 & 0 & 0 & 1 & 0 & 0 & 2 & $0.433237156102395 \mathrm{E}+01$ & $0.278286578531691 \mathrm{E}+01$ & $0.112727477282094 \mathrm{E}+01$ \\
\hline 28 & 0 & 0 & 0 & 1 & 0 & 0 & $0.221163211952812 \mathrm{E}+01$ & $0.498938978770980 \mathrm{E}+01 \mathrm{C}$ & $0.647628325318278 \mathrm{E}+00$ \\
\hline
\end{tabular}


TABLE III: Semi-exponential radial basis functions for $N=40$ with optimized exponents. This wave function produces the total energy $E=-7.47805413551$ a.u. for the ground $1^{2} S$-state of the ${ }^{\infty} \mathrm{Li}$ atom. Only one electron spin-function $\chi_{1}=\alpha \beta \alpha-\beta \alpha \alpha$ was used in these calculations.

\begin{tabular}{|c|c|c|c|c|c|c|c|c|c|c|}
\hline$N$ & & $n_{2}$ & $r$ & & & & $m_{3}$ & $\alpha$ & $\beta$ & $\gamma$ \\
\hline 1 & 0 & 0 & 0 & 0 & & 0 & 1 & $0.309846257882910 \mathrm{E}+01$ & $0.291892609218063 \mathrm{E}+01$ & $0.845633720338414 \mathrm{E}+00$ \\
\hline 2 & 0 & 0 & 0 & 1 & & 0 & 1 & $0.190473561336185 \mathrm{E}+01$ & $0.366143431469990 \mathrm{E}+01$ & $0.157818362413849 \mathrm{E}+01$ \\
\hline 3 & 0 & 0 & 0 & 1 & & 1 & 1 & $0.278598083293758 \mathrm{E}+01$ & $0.282092658543858 \mathrm{E}+01$ & $0.679821359567157 \mathrm{E}+00$ \\
\hline 4 & 0 & 0 & 0 & 2 & & 0 & 1 & $0.288166643409964 \mathrm{E}+01$ & $0.284028201233856 \mathrm{E}+01$ & $0.651027214862940 \mathrm{E}+00$ \\
\hline 5 & 0 & 0 & 1 & 0 & & 0 & 1 & $0.294108078850332 \mathrm{E}+01$ & $0.281478615927614 \mathrm{E}+01$ & $0.660626319649503 \mathrm{E}+00$ \\
\hline 6 & 0 & 0 & 2 & 0 & & 0 & 1 & $0.354836463958720 \mathrm{E}+01$ & $0.298528920333113 \mathrm{E}+01$ & $0.672210253693381 \mathrm{E}+00$ \\
\hline 7 & 0 & 0 & 0 & 0 & & 0 & 0 & $0.287776340439759 \mathrm{E}+01$ & $0.309168157398350 \mathrm{E}+01$ & $0.732676012007879 \mathrm{E}+00$ \\
\hline 8 & 1 & 0 & 0 & 0 & & 0 & 0 & $0.126827836395763 \mathrm{E}+01$ & $0.388838466426963 \mathrm{E}+01$ & $0.696918159081991 \mathrm{E}+00$ \\
\hline 9 & 0 & 0 & 0 & 0 & & 0 & 2 & $0.310409040480143 \mathrm{E}+01$ & $0.297686082083507 \mathrm{E}+01$ & $0.712624162776256 \mathrm{E}+00$ \\
\hline 10 & 1 & 0 & 0 & 0 & & 1 & 0 & $0.304901894182288 \mathrm{E}+01$ & $0.222521841071977 \mathrm{E}+01$ & $0.707931864133508 \mathrm{E}+00$ \\
\hline 11 & 0 & 0 & 3 & 0 & & 0 & 1 & $0.410055124696712 \mathrm{E}+01$ & $0.320833161427739 \mathrm{E}+01$ & $0.685502306391946 \mathrm{E}+00$ \\
\hline 12 & 1 & 0 & 0 & 0 & & 0 & 1 & $0.319281393757494 \mathrm{E}+01$ & $0.245033872966999 \mathrm{E}+01$ & $0.131890477152115 \mathrm{E}+01$ \\
\hline 13 & 0 & 0 & 0 & 0 & & 0 & 3 & $0.212220234455464 \mathrm{E}+01$ & $0.270066283806003 \mathrm{E}+01$ & $0.102980902194010 \mathrm{E}+01$ \\
\hline 14 & 0 & 0 & 1 & 1 & & 0 & 1 & $0.385753710808391 \mathrm{E}+01$ & $0.320997710808722 \mathrm{E}+01$ & $0.149747053647776 \mathrm{E}+01$ \\
\hline 15 & 0 & 0 & 0 & 3 & & 0 & 1 & $0.290829017069616 \mathrm{E}+01$ & $0.343736715561301 \mathrm{E}+01$ & $0.656999154522055 \mathrm{E}+00$ \\
\hline 16 & 0 & 0 & 4 & 0 & & 0 & 1 & $0.762940571197015 \mathrm{E}+01$ & $0.406380788113988 \mathrm{E}+01$ & $0.643367162248368 \mathrm{E}+00$ \\
\hline 17 & 0 & 0 & 0 & 2 & & 2 & 1 & $0.337538643016389 \mathrm{E}+01$ & $0.290752238041757 \mathrm{E}+01$ & $0.757319995998642 \mathrm{E}+00$ \\
\hline 18 & 0 & 0 & 1 & 1 & & 1 & 1 & $0.338067126368468 \mathrm{E}+01$ & $0.336098394507054 \mathrm{E}+01$ & $0.689553038540639 \mathrm{E}+00$ \\
\hline 19 & 0 & 0 & 1 & 2 & & 0 & 1 & $0.376783902187767 \mathrm{E}+01$ & $0.307834289691412 \mathrm{E}+01$ & $0.692428594575284 \mathrm{E}+00$ \\
\hline 20 & 0 & 0 & 1 & 3 & & 0 & 1 & $0.302269266874480 \mathrm{E}+01$ & $0.371054683906740 \mathrm{E}+01$ & $0.259164988133888 \mathrm{E}+01$ \\
\hline 21 & 2 & 0 & 0 & 0 & & 0 & 0 & $0.174071943396373 \mathrm{E}+01$ & $0.316379880955191 \mathrm{E}+01$ & $0.135613707594630 \mathrm{E}+01$ \\
\hline 22 & 1 & 1 & 0 & 0 & & 0 & 0 & $0.196285150420498 \mathrm{E}+01$ & $0.280066377711626 \mathrm{E}+01$ & $0.135193158043399 \mathrm{E}+01$ \\
\hline 23 & 1 & 0 & 0 & 0 & & 2 & 0 & $0.308970260481265 \mathrm{E}+01$ & $0.294746895743190 \mathrm{E}+01$ & $0.815386844474244 \mathrm{E}+00$ \\
\hline 24 & 1 & 0 & 0 & 1 & & 1 & 0 & $0.251712242684944 \mathrm{E}+01$ & $0.408949306371305 \mathrm{E}+01$ & $0.114312512643339 \mathrm{E}+01$ \\
\hline 25 & 0 & 0 & 0 & 0 & & 0 & 4 & $0.321334220677367 \mathrm{E}+01$ & $0.364406360008282 \mathrm{E}+01$ & $0.854918812691407 \mathrm{E}+00$ \\
\hline 26 & 0 & 0 & 1 & 0 & & 0 & 0 & $0.690169324304682 \mathrm{E}+01$ & $0.367442145267222 \mathrm{E}+01$ & $0.515986692127293 \mathrm{E}+00$ \\
\hline 27 & 0 & 0 & 1 & 0 & & 0 & 2 & $0.719809975272803 \mathrm{E}+01$ & $0.307482033524797 \mathrm{E}+01$ & $0.136078304480511 \mathrm{E}+01$ \\
\hline 28 & 0 & 0 & 0 & 1 & & 0 & 0 & $0.363754290732346 \mathrm{E}+01$ & $0.256273214565107 \mathrm{E}+01$ & $0.168423311171287 \mathrm{E}+01$ \\
\hline 29 & 0 & 0 & 0 & 1 & & 0 & 2 & $0.356701987266624 \mathrm{E}+01$ & $0.340559537814167 \mathrm{E}+01$ & $0.824959104823405 \mathrm{E}+00$ \\
\hline 30 & 1 & 0 & 1 & c & & 0 & 0 & $0.244720102707966 \mathrm{E}+01$ & $0.271840294504025 \mathrm{E}+01$ & $0.895838653318275 \mathrm{E}+00$ \\
\hline 31 & 2 & 0 & 0 & c & & 1 & 0 & $0.315627784159638 \mathrm{E}+01$ & $0.302366248489895 \mathrm{E}+01$ & $0.903526045564038 \mathrm{E}+00$ \\
\hline 32 & 1 & 0 & 0 & c & & 1 & 1 & $0.325159760628385 \mathrm{E}+01$ & $0.330340729374998 \mathrm{E}+01$ & $0.897876831570133 \mathrm{E}+00$ \\
\hline 33 & 3 & 0 & 0 & 0 & & 0 & 0 & $0.338532643878470 \mathrm{E}+01$ & $0.298984569514637 \mathrm{E}+01$ & $0.936601520312646 \mathrm{E}+00$ \\
\hline 34 & 2 & 0 & 0 & c & & 0 & 1 & $0.348486218895562 \mathrm{E}+01$ & $0.311367325997526 \mathrm{E}+01$ & $0.975295713010522 \mathrm{E}+00$ \\
\hline 35 & 0 & 0 & 5 & c & & 0 & 1 & $0.575970395842301 \mathrm{E}+01$ & $0.393521634949650 \mathrm{E}+01$ & $0.781294134452016 \mathrm{E}+00$ \\
\hline 36 & 0 & 0 & 0 & 4 & & 0 & 1 & $0.352212817969061 \mathrm{E}+01$ & $0.366200645034756 \mathrm{E}+01$ & $0.347518212792864 \mathrm{E}+01$ \\
\hline 37 & 0 & 0 & 1 & 4 & & 0 & 1 & $0.384658211093920 \mathrm{E}+01$ & $0.412493146244247 \mathrm{E}+01$ & $0.976334599279947 \mathrm{E}+00$ \\
\hline 38 & 0 & 0 & 0 & 5 & & 0 & 1 & $0.359131298628222 \mathrm{E}+01$ & $0.426731681125525 \mathrm{E}+01$ & $0.125620739151171 \mathrm{E}+01$ \\
\hline 39 & 0 & 0 & 2 & 1 & & 0 & 1 & $0.294548846533642 \mathrm{E}+01$ & $0.329899178470137 \mathrm{E}+01$ & $0.769541352183112 \mathrm{E}+00$ \\
\hline 40 & 0 & 0 & 2 & 2 & & 0 & & & $0.454562709500504 \mathrm{E}+01$ & $0.894468554048998 \mathrm{E}+00$ \\
\hline
\end{tabular}

